\title{
Real-time numerical simulation of storm surge inundation using high-performance computing for disaster management, Queensland
}

\author{
Joanna Burston, Takehiko Nose and Rodger Tomlinson \\ Griffith Centre for Coastal Management, Griffith University, Southport, QLD 4215 Australia. \\ t.nose@griffith.edu.au
}

\begin{abstract}
Storm surge presents the greatest hazard to life in tropical cyclone (TC) events, and the Queensland coastline has particular vulnerability to such extreme events. Presently, the Australian Bureau of Meteorology (BOM) issues warnings of forecast storm surge magnitudes using a static look-up table of scenarios associated with forecast cyclone track parameters. In association with Emergency Management Queensland (EMQ), Griffith University has initiated a research project investigating the potential to dynamically model storm surge inundation in real-time during TC events. The project aims to optimise emergency planning and evacuation strategies for storm surge impacts for impending TC landfall by providing outputs at greater spatial resolution, extension into inundation modelling over land, and development of a probabilistic approach to storm tide simulation that may provide emergency managers with a more complete view of possible outcomes.
\end{abstract}

Here we present an overview of a feasible systems approach to real-time storm surge forecasting optimised for run-time, including generation of an ensemble of forcing wind and pressure fields, a hydrodynamic model and post-processing of model output for delivery to emergency management agencies. An ensemble approach to TC forecasting suitable for storm surge modelling has been developed in order to gain an understanding of the critical effects of the spatial, temporal and intensity uncertainties in TC forecasts on the resultant storm surge forecast. Parametric wind/pressure fields for each ensemble member are generated to force the finite volume hydrodynamic model, which has been built using MIKE21 by DHI software implemented on Griffith University and project partner Queensland Cyber Infrastructure Foundations (QCIF)'s high performance computing facilities. Two versions of the hydrodynamic model have been constructed, calibrated and validated for TC storm surge: the 'nearshore' model with spatial resolution of $\sim 1-2 \mathrm{~km}$ in the nearshore zone outputting discrete storm surge magnitudes, and the 'inundation model' with greater spatial resolution capable of dynamically modelling storm tide propagation over land. The output of the nearshore model would be combined with a bath-tub mapping approach to yield probabilistic storm tide inundation estimates. The inundation modelling yields storm tide depth surfaces directly, to be combined into a probabilistic surface.

A case study comparing the simulation time required to model storm tide inundation using the two approaches for TC Yasi is presented. The nearshore modelling combined with the bath-tub mapping approach yields achievable run-times for an ensemble forecast. The inundation modelling approach is currently too computationally expensive for the ensemble forecasting method developed for this study and efforts to optimise the simulation time are underway. A comparison of the resultant storm tide inundation levels obtained by each approach is in progress. Understanding these differences may lead to the development of techniques to improve the bath-tub mapping approach.

Keywords: Storm surge, tropical cyclone, numerical modelling, high performance computing, emergency management. 
Burston, Nose and Tomlinson, Real-time numerical simulation of storm surge inundation using highperformance computing for disaster management, Queensland.

\section{INTRODUCTION}

Elevated ocean water levels due to storm surge in combination with the astronomical tide (referred to here as storm tide) experienced during a tropical cyclone (TC) event pose a significant hazard to human life, in combination with high flow velocities, severe wind conditions and wave effects. The Queensland coastline has high exposure to this hazard, with 207 known impacts from tropical cyclones along the east coast since 1858 (BOM, 2009), combined with vulnerability of low-lying and growing coastal population centres. Recent tropical cyclone events generating hazardous storm surge include TC Yasi (2011) and TC Larry (2006), both impacting the Mission Beach area in Far North Queensland. The major population centres of Cairns, Townsville and Mackay are all vulnerable to storm tide events. This research project in partnership with EMQ, QCIF and DHI Australia investigates the potential for real-time forecasting of storm surge inundation during TC events using numerical modelling for the purposes of emergency management.

The current emergency management response to an impending storm tide hazard in Queensland is evacuation to higher ground, with effective evacuation requiring 24 to 48 hours of lead time prior to the onset of gale force winds. EMQ and local councils use Storm Tide Warning advice issued by BOM to determine the need for evacuations. BOM's current Storm Tide Warning system is deterministic in nature, and consists of matching the consensus forecast TC track to a library of pre-run storm surge simulations made using a hydrodynamic model forced with parametric cyclonic wind fields (Harper et al., 2001). The resultant storm surge heights, output at widely-spaced discrete locations along the coastline, are then added to the highest tide of the day until $\sim 12-24$ hours prior to landfall. As certainty in TC landfall time and location develops, the appropriate predicted tide magnitude is used (pers. comm., B. Gunn).

A stakeholder engagement program with disaster managers at EMQ and coastal local councils has helped us identify some concerns with the current Storm Tide Warning system and a range of potential useful improvements. Two key improvements of higher spatial resolution and probabilistic output are discussed further here. Firstly, the current sparsely-dispersed warning sites along the Queensland coastline makes interpretation of potential onshore inundation due to storm tide difficult, and modelling at higher spatial resolution giving more continuous spatial output is desirable. Currently, EMQ use the discrete water levels given in BOM's Storm Tide Warnings to create surfaces corresponding to the estimated storm tide levels in Geographical Information System (GIS). In order to identify pre-defined evacuation zones, these surfaces are intersected with the terrestrial Digital Elevation Model (DEM) (pers. comm.. J. Arrowsmith). However, this bath-tub inundation approach neglects several dynamical features of storm surge propagation, including longshore variations due to local bathymetry, the higher onshore storm surge inundation level compared to that in the nearshore owing to wind set-up as well as the behaviour of storm surge in estuaries. To address this need, we propose an extension of storm surge simulation to inundation modelling using a finite volume flexible mesh hydrodynamic model.

The need for a probabilistic understanding of storm tide risk TC forecasting is critical given the high uncertainties in forecast TC track and intensity. The uncertainties in the forecast TC track mean that the location of landfall and hence peak storm surge can vary by hundreds of kilometres 48 hours prior to landfall, but the current deterministic warning system does not give emergency managers a view of these uncertainties. The uncertainty with regard to timing of landfall means that there is a large range in the potential storm tide magnitude depending on the timing of peak storm surge with the astronomical tide. The uncertainties in TC intensity (peak wind speed) are also critical as storm surge magnitude varies as a function of the square of the wind speed. The deterministic nature of the current Storm Tide Warning system means that emergency managers do not currently have a view of the range of storm tide scenarios that could result from a given point in the forecast. Here we have developed a probabilistic approach to storm tide simulation driven by an ensemble of TC forcing, which could give probabilistic guidance on potential storm tide inundation risk in a real-time event.

Taking on board the findings of the stakeholder consultations, we present here a feasible system for real-time storm tide inundation forecasting using dynamic wind and ocean models outputting high resolution probabilistic estimates of inundation along the eastern Queensland coast and outline the computational resources required to implement such a system. The real-time system is also designed to meet a requirement for forecast turn-around within one hour of TC track updates. The modelling framework, which uses TC track information available to Australian forecasters as input, is presented in Section 2. A case study for TC Yasi is then given in Section 3. 
Burston, Nose and Tomlinson, Real-time numerical simulation of storm surge inundation using highperformance computing for disaster management, Queensland.

\section{PROPOSED STORM TIDE FORECASTING SYSTEM}

The real-time storm tide forecasting system consists of: (1) a method to select a probabilistic ensemble of TC events suitable for simulating storm tide based on TC intensity, scale, and track (landfall location and timing); (2) an extension of existing wind field generation techniques using TC parameter information available in real-time; (3) a calibrated and validated hydrodynamic model optimised for real-time forecasting with the option of either nearshore storm tide output or inundation modelling; enabling delivery of probabilistic inundation maps to emergency managers. Each of these components is discussed in further detail below.

\subsection{TC Track Ensemble Selection}

The size of the TC ensemble necessary to capture the range of storm surge possibilities at a given point in a forecast informs subsequent development of the wind field and hydrodynamic modelling framework by providing guidance on the required trade-off between the spatial resolution of the model and computational resources necessary to complete such simulations within the desired timeframe. Ascertaining the size and composition of this TC ensemble requires an understanding of the sensitivities of the predicted storm surge to the various input parameters defining the TC wind fields.

In terms of available probabilistic TC information in forecast mode, BOM operationally generate a 1000member stochastic set of tropical cyclone tracks for the purposes of generating wind hazard probability maps (pers. comm.., Dr M. Foley, 2013). This set of tracks is based on the consensus forecast track, and created using the method of DeMaria et al. (2009) by sampling the errors in cyclone parameters from distributions computed from the errors between the consensus and ultimate best track at different forecast periods for cyclone events in the Australian region over a five year period. A sample of this ensemble for TC Yasi at 48 hours from landfall is shown in Figure 1. A method to reconstitute a set of representative tracks from the probability distributions of the parameters important for storm surge (TC landfall location, intensity, and scale) derived from this 1000-member ensemble with associated probabilities of occurrence has been developed for this study for use in real-time probabilistic storm surge simulation (Burston et al., 2013). The ensemble tracks are recomputed every forecast output period. As the cyclone progresses closer to the coastline and the range of landfall locations narrows, the spacing between landfall locations can be decreased, which would give a more detailed view of the resultant probabilistic inundation surfaces.
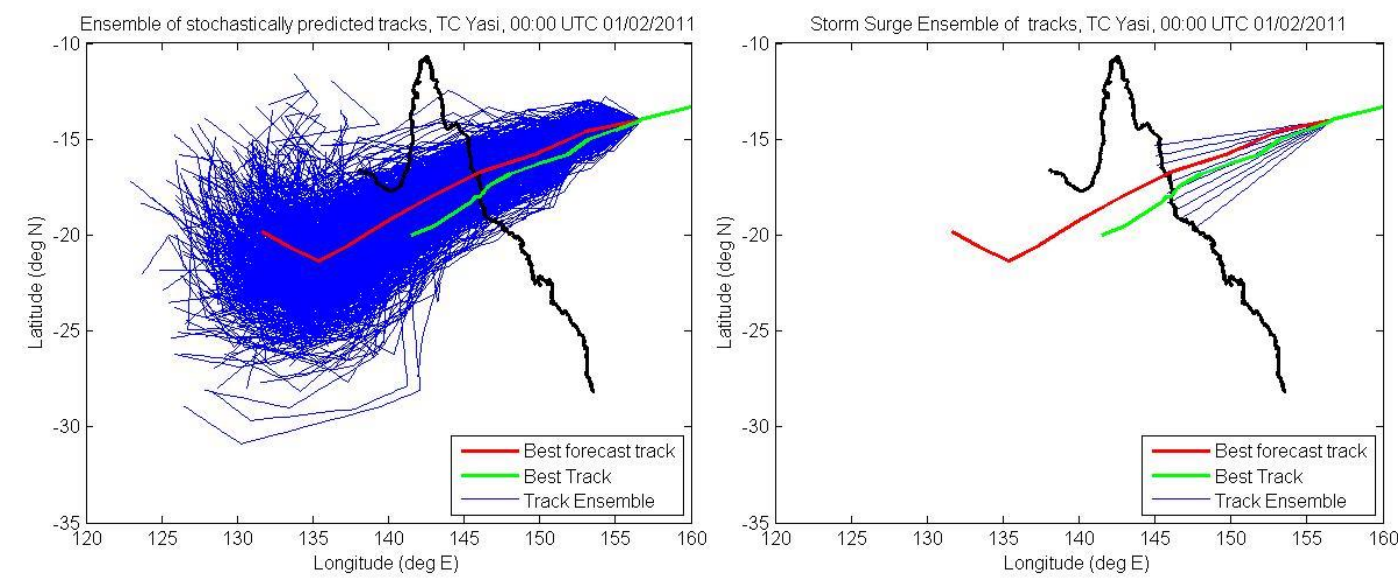

Figure 1 Example of the DeMaria 1000-member TC track ensemble for TC Yasi at 48 hours from landfall, showing considerable spatial spread in potential landfall locations along the Queensland coast (black) (courtesy M. Foley, Centre for Australian Weather and Climate Research) (left), and the resultant 282member TC ensemble for storm surge simulation (each track has a range of associated intensity and scale parameters) (right).

\subsection{Wind field generation}

Modelled surface wind and atmospheric pressure fields are the forcing required for hydrodynamic simulation of storm surge. Current operational wind field models are not suitable for use as forcing for storm surge modelling as their current spatial resolution is inadequate to capture the peak intensity of the TC and in forecast mode, the TC intensity and track are not predicted accurately enough for the needs of storm surge modelling. Instead, parametric techniques such as those formulated by Holland (1980), Holland et al. (2010) and others are widely applied to provide forcing for storm surge modelling, including forming the basis for the current storm surge forecast modelling for Queensland (Harper et al., 2001). Such parametric 
Burston, Nose and Tomlinson, Real-time numerical simulation of storm surge inundation using highperformance computing for disaster management, Queensland.

formulations use forecast information on TC track, central and ambient pressure, radius to maximum winds (RMW) and a scaling factor (e.g., Holland's B) as input and rely on the relative uniformity of cyclonic circulations in order to simulate cyclonic wind and pressure fields (Holland et al., 2010).

In order to generate wind and pressure field inputs for storm surge modelling for each TC ensemble member, an extension of the Holland et al. (2010) (H10) single vortex wind profile has been implemented. The radial atmospheric pressure/wind speed model is extended to time and azimuth dimensions, and the radius to gales is fixed using the average of the R34 quadrant values given in the DeMaria ensemble. The H10 wind profile model has an advantage over the presently used Holland (1980) model by reducing underestimation of the wind speeds in the tails of the wind profile and also reducing sensitivity to input RMW. This wind field model was found to well reproduce the measured storm surge from TC Yasi and other historical events when used to force the hydrodynamic model described below (Burston, 2013).

\subsubsection{Storm surge hydrodynamic model - MIKE21 FM}

A calibrated and validated hydrodynamic model optimised for real-time forecasting of storm surge inundation has been established using DHI's MIKE21 finite volume hydrodynamic model for high performance computing (HPC) implemented on Griffith University and QCIF's HPC facilities. Use of a finite volume model facilitates implementation of the complex landward boundary condition required for inundation modelling. The unstructured triangular mesh elements enable focusing of spatial resolution in areas with high storm tide risk and those that are hydrodynamically complex while minimising excess runtime and negating any need for nesting. The model is 2D depth-integrated and includes wetting and drying.

The model bathymetry is the best available as sourced from multiple local, state, and federal agencies and industry, and adjusted to AHD (Figure 2). The land elevation was obtained from 1-m LiDAR data supplied by the Queensland State government. The model has been calibrated to the astronomical tide for 13 measurement sites from the Gold Coast Seaway (27.95 ${ }^{\circ} \mathrm{S}$ ) to Cooktown (15.45 ${ }^{\circ} \mathrm{S}$ ) (Burston and Symonds, 2013). Refinement of the Great Barrier Reef was necessary in order to capture the complex tidal flows through various channels. Two variations of the model have been developed. The 'nearshore' model does not extend onto land and outputs storm surge estimates in the nearshore where mesh elements are approximately $1 \mathrm{~km}$ across except where finer detail is required in channels and around islands. The 'inundation' model extends onshore to the estimated maximum upper inundation level with higher spatial resolution of up to 30 metres. The finer inundation model has been established at this stage for the test location of Cassowary Local Council area, being the impact site for TCs Yasi and Larry with supporting calibration data. The finer 'inundation' model takes much longer to run, as detailed in the case study below.
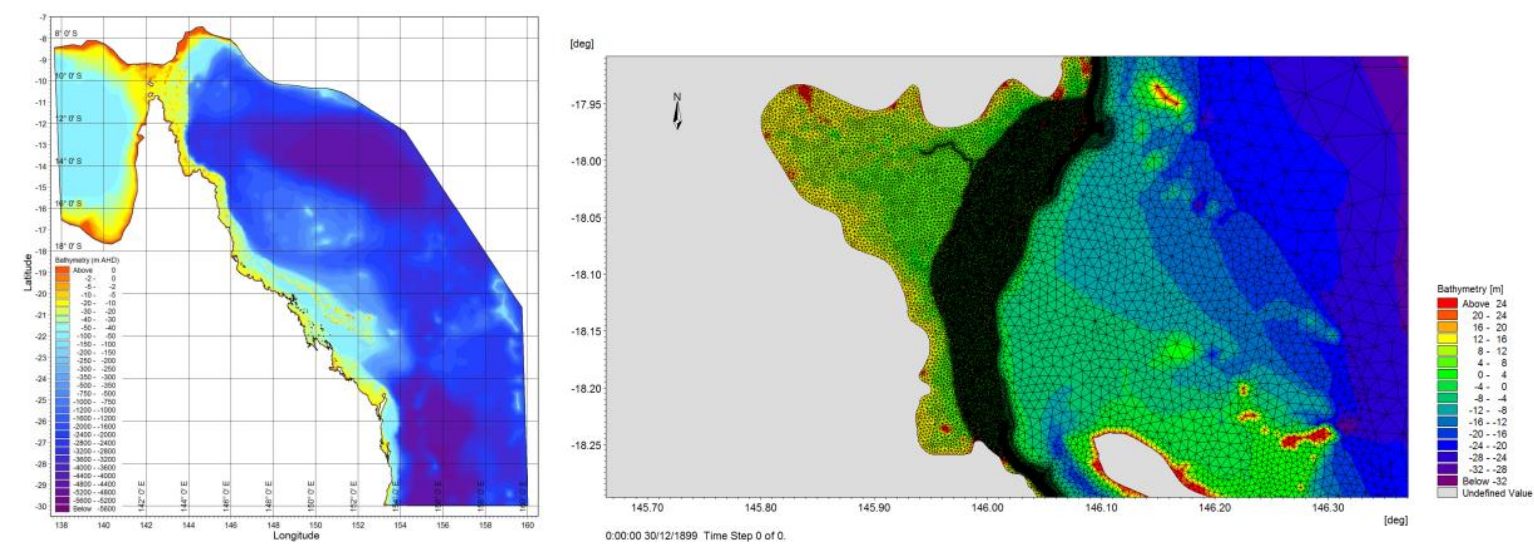

Figure 2 Extent of the Coral Sea 'nearshore' model and bathymetry (left) and a close-up of the 'inundation' model mesh in the Cardwell area showing the variable spatial resolution (right).

Effort was spent optimising the model mesh for real-time performance, achieving a $50 \%$ reduction in runtime. Benchmarking shows close to linear speed up of run-time to 32 cores on Griffith's HPC for tidal simulations. A comparison of modelled to measured tidal constituents shows good agreement in both amplitude and phasing. Validations of storm surge levels for several historical events including TC Yasi (2011) and TC Larry (2006) showed that the 'nearshore' model is capable of replicating the peak storm surge levels measured at storm tide gauges (Burston, 2013) and that the 'inundation' model is capable of replicating the measured extent of inundation due to TC Yasi. Run-time of the hydrodynamic model is optimised for each TC ensemble member simulation by selecting an appropriate domain extent within the 
Burston, Nose and Tomlinson, Real-time numerical simulation of storm surge inundation using highperformance computing for disaster management, Queensland.

greater Coral Sea model. A pre-defined library of meshes has been created and a tool to select the appropriate simulation domain based on the forcing TC area of influence developed.

\subsubsection{Storm tide inundation mapping}

Here we discuss two alternatives to yield the required output of probabilistic storm tide inundation maps, the first using the coarser 'nearshore' model with faster run-time combined with a bath-tub inundation approach, and the second using the finer but slower 'inundation' model which dynamically maps storm tide propagation over land. The proposed approaches for each alternative are outlined below.

\subsubsection{Bath-tub mapping approach}

Using the 'nearshore' model, storm surge water level at discrete output points located at a spacing 1-2 km along the eastern coastline of Queensland in populated areas are output for each TC ensemble member. Simulations are made on a constant initial water level (without a tide) given the uncertainty in forecast TC track timing. Using the calibrated Coral Sea tidal model, a predicted tide time series for each model output point is generated. For the standard port sites, the measured tidal constituents are used to predict the tides. For each TC ensemble member, the TC landfall time is used to select the corresponding tidal water level at each output point. The empirical probability distribution of the tidal water level at each output point is then calculated from which $n_{t}$ realisations are drawn and linearly combined with the storm surge ensemble results.

Probabilistic storm tide heights at each model output point are calculated using the probabilities associated with each TC ensemble member. These probabilities can be expressed either as the probability of storm tide greater than $X$ metres, or storm tide magnitude $(\mathrm{m})$ that is exceeded by $Y \%$ of ensemble members (as for National Oceanic and Atmospheric Administration's (NOAA) p-surge model; Taylor and Glahn, 2008). Both outputs were identified as useful for disaster managers.

The resultant probabilistic storm tide heights would then be delivered to EMQ to map predicted inundation using a bath-tub approach in GIS software. A storm tide surface for each probability level is created by interpolating between the model output points, and then intersected with the terrestrial DEM to calculate inundation depths. The resultant probabilistic inundation depth extents and surfaces can then be intersected with the pre-defined evacuation zones by EMQ and local emergency managers to identify areas requiring evacuation orders.

Although this approach has similar disadvantages to the present BOM Storm Tide Warning modelling in that it does not account for dynamic propagation of storm tide over land, it is an improvement in that the spatial variation in storm surge is more finely resolved than at present, where the estimated storm tide output sites used in the bathtub approach are widely spaced and the storm tide magnitudes discontinuous.

\subsubsection{Inundation modelling approach}

Using the finer 'inundation' model, storm tide water levels over land are modelled explicitly, outputting an unstructured grid of storm tide surface elevations. Probabilistic storm tide heights are then calculated similar to that outlined in approach (Section 2.3.2.1). The resultant values would then be imported into a GIS by EMQ, interpolated and intersected with the LiDAR elevation DEM to define probabilistic inundation depths and extents.

While this method presents advantages in terms of capturing the dynamic behaviour of storm tide over land and in estuaries, it requires a greater number of simulations due to the inclusion of the tidal phases as a variable in the ensemble set and much greater simulation time given the high resolution model mesh.

This project seeks to identify whether the increased accuracy afforded by the second approach warrants the additional investment in computational resources. Investigation into the differences in storm tide inundation prediction between the bath-tub approach and the dynamical approach is continuing. Some preliminary results for a case study on TC Yasi are presented in the next section.

\section{TC YASI CASE STUDY}

TC Yasi (2011), impacting the Cassowary Local Council area in North Queensland, is used as a case study testing the end-to-end storm tide forecasting system with the two proposed options discussed above. We examine the trade-off between the spatial resolution of the hydrodynamic model and the computational resources that would be required to simulate storm surge inundation with the proposed ensemble approach. The resulting TC ensemble for TC Yasi 48 hours prior to landfall contains 282 members, assuming landfall locations at 0.5 degree intervals along the coastline. In terms of operational run-time, the maximum allowable simulation time (MAST) each hydrodynamic simulation would be required to run in $\left(X^{*} 45^{*} 60\right) / n$ seconds 
Burston, Nose and Tomlinson, Real-time numerical simulation of storm surge inundation using highperformance computing for disaster management, Queensland.

per core for $X$ computing cores (e.g., 282 simulations, MAST $=612$ sec per run per core for 64 cores, MAST $=1224 \mathrm{sec}$ per run per core on 128 cores) in order to achieve a one-hour turn-around from each TC track forecast update assuming $45 \mathrm{~min}$ for simulation and $15 \mathrm{~min}$ for pre- and post-processing.

Starting with the 'nearshore' model, we have found a model run-time of $130 \mathrm{sec}$ per core per ensemble member for TC Yasi for the model domain shown in Figure 3 extending from north of Cairns to south of Townsville. This model resolution enables accurate simulation of the peak measured storm surge height along the extent of the Queensland coastline affected by this event (Figure 4). This model run-time would meet the ensemble turn-around requirements discussed above with less than 16 computational cores.

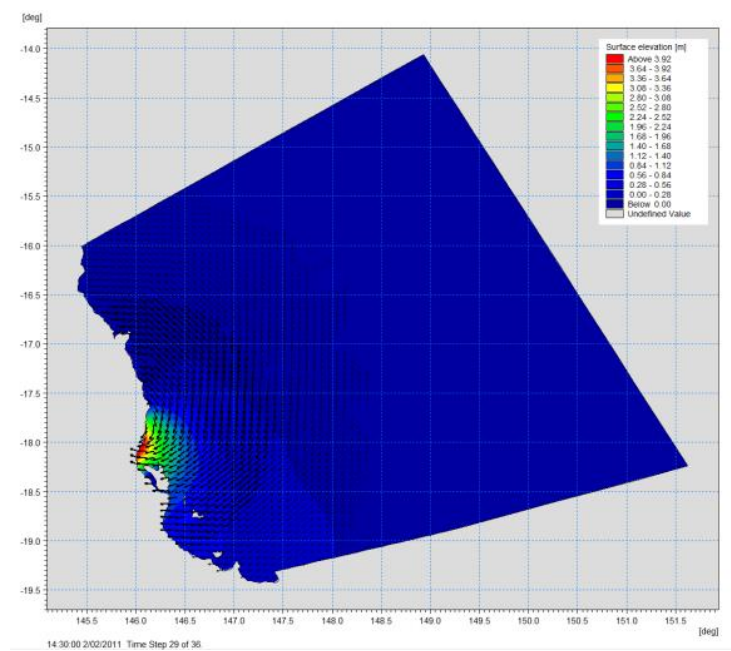

Figure 3 Sample model domain for TC Yasi from Townsville to Cairns showing the storm surge water level at landfall using the best validated Holland et al. (2010) wind/pressure field.
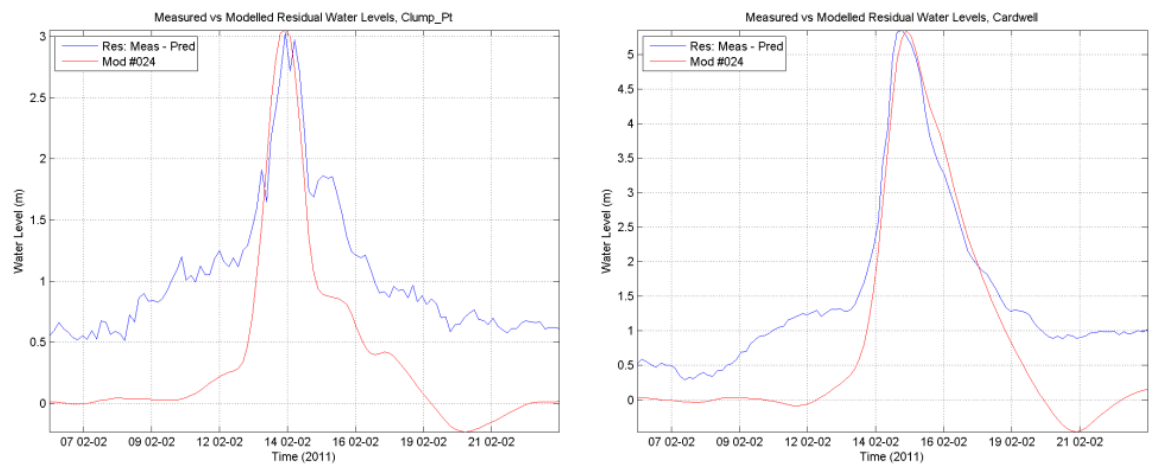

Figure 4 Comparison of modelled vs measured storm surge at Clump Point (left) and Cardwell (right) for TC Yasi using the 'nearshore' model forced with a Holland et al. (2010) wind field. Measured storm tide gauge data courtesy of Maritime Services Queensland.

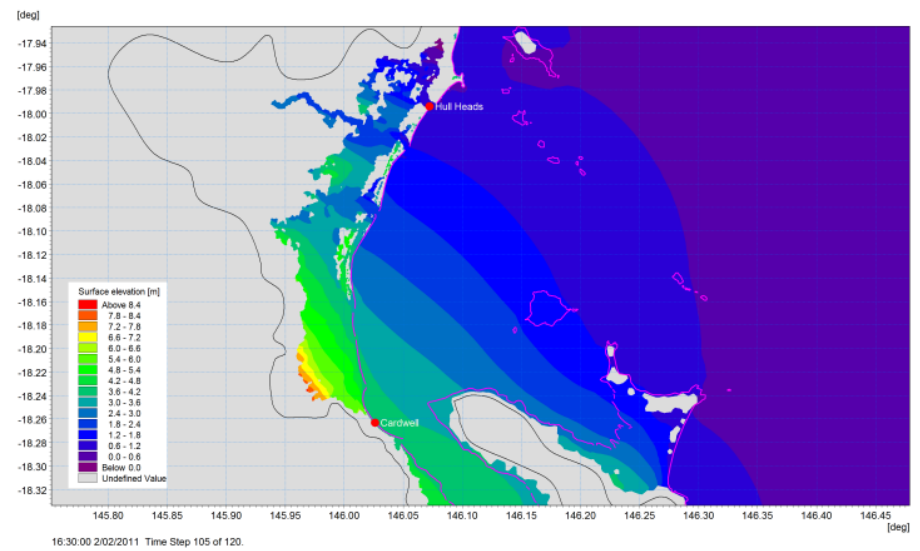

Figure 5 Sample results of the inundation model for TC Yasi in the Tully Beach to Cardwell area. 
Burston, Nose and Tomlinson, Real-time numerical simulation of storm surge inundation using highperformance computing for disaster management, Queensland.

We found through the model validation process that mesh elements with a spatial resolution of up to 30 metres are required to replicate the storm surge inundation over land observed due to TC Yasi (Figure 5). The inundation model at this resolution covering coastal townships in the Cassowary Local Government area contains 99535 elements. The simulation time is $4912 \mathrm{sec}$ per core per ensemble member (38 times the length of the coarser model). This particular model runs most efficiently across 16 cores. A full ensemble set including three variations of the tidal phasing would require computational resources of $(4912 * 282 * 3 /(45 * 60)=) 1539$ cores to achieve the required operational turn-around. This would be expected to be higher for more populated risk areas where the inundation model would contain more mesh elements.

\section{SUMMARY}

Here we have described feasible options for probabilistic real-time forecasting of storm tide and mapping of inundation based on the requirements of emergency managers for higher spatial resolution output and probabilistic understanding of storm tide hazard. The system involves: construction of a TC ensemble suitable for storm surge simulation based on available forecast information, a parametric wind/pressure model, a 2D-depth integrated finite volume hydrodynamic model and either a bath-tub mapping technique or high-resolution inundation modelling to output storm tide depths over land. The final output would be probabilistic maps of potential storm tide inundation hazard.

The optimised hydrodynamic model has been established and tested on high performance computing. From a case study of TC Yasi, the 'nearshore' version of the model outputting storm surge estimates in the nearshore yields run-times for TC events suitable for this ensemble modelling approach. The inundation modelling approach currently has prohibitive simulation times for an ensemble approach, especially as more than one council area may be affected by a given event. Efforts are underway to optimize the inundation model runtime by focusing the model resolution. In addition, a comparison of the resultant storm tide inundation levels obtained by each approach in order to measure the value added by the inundation modelling is in progress. Understanding these differences may lead to the development of techniques to improve the bath-tub mapping approach. Additional inundation due to wave setup also remains an outstanding issue for future work.

\section{REFERENCES}

Bureau of Meteorology. 2009. Tropical Cyclones in Queensland. http://www.bom.gov.au/cyclone/about/eastern.shtml

Burston, J.M. 2013. Coral Sea Hydrodynamanic Model Storm Surge Validation Report. Technical Report 142. Griffith Centre for Coastal Management.

Burston, J.M. and Symonds, A.M. 2013. Coral Sea Hydrodynamanic Model Set-up and Calibration. Technical Report 140. Griffith Centre for Coastal Management.

Burston, J.M., Symonds, A.M. and Tomlinson, R. 2013. Options for real-time storm tide forecasting for emergency management in Queensland, Australia. Proceedings of Coasts and Ports 2013. Sydney. Sept 2013.

DeMaria, M., Knaff, J.A., Knabb R., Lauer, C., Sampson, C.R., DeMaria, R.T. 2009. A new method for estimating tropical cyclone wind speed probabilities. Weather and Forecasting 24.6:1573-1591.

Harper, B.A., Hardy, T.A., Mason, L.B., Bode, L., Young, I.R. and Nielsen, P. 2001. Queensland climate change and community vulnerability to tropical cyclones, ocean hazards assessment. Stage 1 report. Department of Natural Resources and Mines, Queensland, Brisbane, Australia, 368 pg.

Holland, G.J., 1980. An analytical model of the wind and pressure profiles in hurricanes. Mon. Wea. Rev. $108,1212-1218$.

Holland, G.J., Belanger, J.I., and Fritz, A. 2010. A revised model for radial profiles of hurricane winds. Mon. Wea. Rev. 138: 4393-4401.

Taylor, A.A., and Glahn, B. 2008. Probabilistic guidance for hurricane storm surge. In: 19th Conference on probability and statistics. Section. 7.4. 